\title{
One-third of Danish people will receive secondary care treatment for a mental disorder during their lifetime
}

\section{Marta Miret}

Department of Psychiatry, Universidad Autónoma de Madrid, Instituto de Salud Carlos III, Centro de Investigación Biomédica en Red de Salud Mental. CIBERSAM, Madrid, Spain; marta.miret@uam.es

\section{WHAT IS ALREADY KNOWN ON THIS TOPIC?}

There is a need to assess the lifetime risks for mental disorders in order to plan healthcare services. Incidence rates of mental disorders have been estimated by age and sex in population-based prospective surveys. Previous studies that have used health registries to estimate incidence and risk have focused on only one specific disorder. ${ }^{1}$ Nevertheless, no nationwide studies that provide comprehensive assessments of lifetime risks for treated mental disorders have been carried out.

\section{WHAT DOES THIS PAPER ADD?}

- This is the first comprehensive nationwide assessment of lifetime risks, sex-specific and age-specific incidence rates and sex-specific and age-specific cumulative incidence rates of mental disorders treated in secondary care during a lifetime.

- The results show that one-third of the Danish population will receive treatment in secondary care for a mental disorder across their lifetime. This finding is close to the lifetime prevalence found in previous community surveys. ${ }^{2}$ Many mental disorders had a single peak during the second and third decades of life, whereas some disorders had a second peak later in life.

- The study complements results from population-based surveys as it was able to obtain information from persons who were institutionalised or homeless, groups that are not usually captured in population-based surveys. The diagnoses were made by a comprehensive clinical assessment, and the results are not influenced by the reporting bias, usually present in population-based surveys.

\section{LIMITATIONS}

- If people with untreated disorders had been included, the estimates would have been significantly higher, since previous studies have estimated that $36-50 \%$ of serious cases with mental disorders in developed countries are untreated. ${ }^{3}$ Mild and moderate mental disorders treated only by general practitioners or by specialists in psychiatry working in private practice were also not included.

- Clinician-derived diagnosis lacks the reliability that can be obtained by well-trained interviewers using standardised diagnostic interviews such as the Composite International Diagnostic Interview. ${ }^{4}$

\section{WHAT NEXT IN RESEARCH}

Further studies are needed to look at the onset of new episodes of illness after the first one (relapse rate), analyse the course of the disorder and obtain information about healthcare utilisation. Useful epidemiological data could be obtained from nationwide mental health registers in other countries, which could allow making comparisons about the performance of different national healthcare systems. The comparison of Pedersen and colleagues' results with the results in less developed countries would be interesting. Nevertheless, it is extremely difficult to carry out similar analysis in many countries due to the absence of nationwide mental health registries representative of the general population.

\section{COULD THESE RESULTS CHANGE YOUR PRACTICES AND WHY?}

Yes. The age distributions can help clinicians know the age at which the incidence of different mental disorders can have their peak, which might be helpful for early detection and prevention of mental disorders. Special attention to patients in their 20s and 30s and those over 60 must be paid, since those are the times at which many disorders have their onset. Furthermore, the results have important implications for planning healthcare services and can guide allocation of future healthcare funding in terms of total demand estimated over the lifetime, the age distributions expected at different treatment facilities and the ages at which primary prevention activities are targeted. The results also show the public health needs of young people and of those over 60.

\section{Competing interests None.}

doi:10.1136/eb-2014-101926

\section{REFERENCES}

1. Thorup A, Waltoft BL, Pedersen CB, et al. Young males have a higher risk of developing schizophrenia: a Danish register study. Psychol Med 2007;37:479-84.

2. Kessler RC, Angermeyer M, Anthony JC, et al. Lifetime prevalence and age-of-onset distributions of mental disorders in the World Health Organization's World Mental Health Survey Initiative. World Psychiatry 2007;6:168-76.

3. Demyttenaere K, Bruffaerts R, Posada-Villa J, et al. Prevalence, severity, and unmet need for treatment of mental disorders in the World Health Organization World Mental Health Surveys. JAMA 2004;291:2581-90.

4. Andrews G, Peters L. The psychometric properties of the Composite International Diagnostic Interview. Soc Psychiatry Psychiatr Epidemiol 1998;33:80-8.

ABSTRACT FROM: Pedersen CB, Mors O, Bertelsen A, et al. A comprehensive nationwide study of the incidence rate and lifetime risk for treated mental disorders. JAMA Psychiatry 2014;71:573-81.

Population All Danish residents born between 1 January 1900 and 31 December 2010. Incident cases were the first contact with secondary care during 2000-2012 for any mental health illness, as recorded in the Danish Psychiatric Central Research Register using ICD codes.

\section{OUTCOMES}

Study characteristics 5.6 million Danish residents were included in the cohort, which was followed up for 59.5 million person years.

Risk for any psychiatric disorder Lifetime risk was 37.66\% (female) and $32.05 \%$ (male). The incidence of any disorder before the age of 50 was $25.26 \%$ (female) and $22.60 \%$ (male).
Risk for schizophrenia-related disorders Lifetime risk was $3.67 \%$ (female) and $3.78 \%$ (male). The incidence before the age of 50 was $2.43 \%$ (female) and $3.06 \%$ (male).

Risk for bipolar affective disorder Lifetime risk was $1.84 \%$ (female) and $1.32 \%$ (male). The incidence before the age of 50 was $1.07 \%$ (female) and $0.76 \%$ (male).

Risk for single and recurrent depressive disorder Lifetime risk was $15.50 \%$ (female) and $9.07 \%$ (male). The incidence before the age of 50 was $10.18 \%$ (female) and $5.63 \%$ (male).

Risk for neurotic, stress-related and somatoform disorders Lifetime risk was $18.97 \%$ (female) and $12.51 \%$ (male). The incidence before the age of 50 was $15.80 \%$ (female) and $10.28 \%$ (male).

Risk for Alzheimer's disease Lifetime risk was 5.14\% (female) and 3.20\% (male). The incidence before the age of 50 was $0.01 \%$ (female) and $0.01 \%$ (male). 